\title{
ON FREE IDEALS IN FREE ALGEBRAS OVER A COMMUTATIVE RING
}

\author{
Khurul Wardati $^{1}$, Indah Emilia Wijayanti ${ }^{2}$, and Sri Wahyuni ${ }^{3}$ \\ ${ }^{1}$ Postgraduate Program, Department of Mathematics, \\ Universitas Gadjah Mada, Yogyakarta, Indonesia \\ ag_khurul@yahoo.co.id \\ ${ }^{2}$ Department of Mathematics, \\ Universitas Gadjah Mada, Yogyakarta, Indonesia \\ ind_wijayanti@ugm.ac.id \\ ${ }^{3}$ Department of Mathematics, \\ Universitas Gadjah Mada, Yogyakarta, Indonesia \\ swahyuni@ugm.ac.id
}

\begin{abstract}
Let $A$ be a free $R$-algebra where $R$ is a unital commutative ring. An ideal $I$ in $A$ is called a free ideal if it is a free $R$-submodule with the basis contained in the basis of $A$. The definitions of free ideal and basic ideal in the free $R$-algebra are equivalent. The free ideal notion plays an important role in the proof of some special properties of a basic ideal that can characterize the free $R$-algebra. For example, a free $R$-algebra $A$ is basically semisimple if and only if it is a direct sum of minimal basic ideals in $A$. In this work, we study the properties of basically semisimple free $R$-algebras.

Key words and Phrases: Free ideal, basic ideal, minimal basic ideal, basically semisimple algebra.

Abstrak. Misalkan $A$ adalah $R$-aljabar bebas dengan $R$ ring komutatif dengan elemen satuan. Ideal $I$ di $A$ disebut ideal bebas jika $I$ merupakan suatu $R$-submodul bebas dengan basis yang termuat dalam basis dari $A$. Definisi ideal bebas dan ideal dasar dalam $R$-aljabar bebas adalah ekuivalen. Ideal bebas berperan penting dalam pembuktian beberapa sifat khusus dari ideal dasar yang dapat mengkarakterisasi $R$-aljabar bebas. Sebagai contoh, suatu $R$-aljabar bebas $A$ adalah semisederhana mendasar jika dan hanya jika $A$ merupakan hasil tambah langsung dari ideal-ideal dasar minimal di $A$. Penelitian ini membahas sifat-sifat semisederhana mendasar dari $R$-aljabar bebas.
\end{abstract}

2000 Mathematics Subject Classification: 16G30.

Received: 17-03-2014, revised: 23-12-2014, accepted: 09-03-2015. 
Kata kunci: Ideal bebas, ideal dasar, ideal dasar minimal, aljabar semisederhana mendasar.

\section{INTRODUCTION}

An $R$-algebra is said to be a free $R$-algebra if it is both, a ring and a free $R$-module [2]. Any algebra over a field is always a free algebra. Throughout this paper, ring $R$ is a unital commutative ring.

Let $K$ be a field and $X$ any basis of $K$-algebra $A^{\prime}$. An arbitrary ideal $I^{\prime}$ in the $K$-algebra $A^{\prime}$ always satisfies the following condition : $k x \in I^{\prime}$ implies $x \in I^{\prime}$, for every non-zero $k \in K$ and $x \in X$. This is due to each non-zero element in $K$ has an inverse. This condition is not always true for an ideal in a free $R$ algebra. As a counter example, consider $M=\left\{(a, b): a, b \in \mathbb{Z}_{6}\right\}$ is a $\mathbb{Z}_{6}$-algebra. Take $M^{\prime}=\left\{(m, n): m, n \in 2 \mathbb{Z}_{6}\right\}$ the ideal in $M$, which does not satisfy the condition. It is because there exists a basis $S=\{(1,0),(0,1)\}$ of $M$, in which $2(1,0)=(2,0) \in M^{\prime}$ but $(1,0) \notin M^{\prime}$. However, the ideal $M^{\prime \prime}=\left\{(a, 0): a \in \mathbb{Z}_{6}\right\}$ in $M$ satisfies the condition, i.e. if $r(1,0)=(r, 0) \in M$ ", then $(1,0) \in M$ " for every non-zero $r \in \mathbb{Z}_{6}$. We define this special ideal as follows. Let $A$ be a free $R$-algebra. An ideal $I$ in $A$ is called a basic ideal, if for every non-zero $r \in R$ and every $x \in X, r x \in I$ then $x \in I$ for any basis $X \subset A$. This term refers to the basic ideal in Leavitt path algebras over unital commutative ring on a graph (see [4]).

We know that an ideal (or subalgebra) of $R$-algebra $A$ is an ideal (or subring) of $A$ and is an $R$-submodule [2]. Not all ideals in a free $R$-algebra have a basis, because not all $R$-submodules in a free $R$-module are free $R$-submodules. From the above example, $M^{\prime}$ does not have a basis, although it is generated by $\{(2,0),(0,2)\}$. However, the ideal $M$ " is an ideal having a basis $\{(1,0)\} \subset S$. Another simple example, let ring $\mathbb{Z}$ can be viewed as free algebra over itself and as a basis is $\{1\}$. The ideal $2 \mathbb{Z}=\langle 2\rangle$ is not a basic ideal in $\mathbb{Z}$. Moreover, the ideal $2 \mathbb{Z}$ is a free $\mathbb{Z}$ submodule in $\mathbb{Z}$ with basis $\{2\} \nsubseteq\{1\}$. This motivates us to define an ideal that has a certain basis. Generally, an ideal in a free $R$-algebra is called a free ideal, if it has a basis contained in a basis of the algebra. We will show that the property of an ideal to be free is necessary and sufficient for being a basic ideal

Based on the basic ideal in Leavitt path algebras over a unital commutative ring studied by Tomforde [4], the further notions are also defined such as minimal basic ideal, prime basic ideal, and semiprime basic ideal. Those notions characterize some properties of Leavitt path algebras, e.g. basically semisimple, basically prime and basically semiprime [5]. Analogously, we show that a minimal basic ideal in a free $R$-algebra will characterize basically semisimple free $R$-algebra.

In this paper, we refer to [7] for a semisimple ring ( or algebra). A semisimple ring (or algebra) is a ring (or algebra) which can be decomposed into a direct sum of its minimal ideals. In addition, an algebra is semisimple if and only if every ideal in the algebra is generated by a central idempotent element ([3], [7]).

Analogous to [5],[6] and [7], a free $R$-algebra $A$ is basically semisimple if it is a direct sum of minimal basic ideals in $A$. The focus of this paper is to prove that 
a free $R$-algebra $A$ is basically semisimple if only if it is a finite sum of minimal basic ideals in $A$ and if only if every basic ideal in $A$ is a direct summand of $A$ and if only if every basic ideal in $A$ is generated by a central idempotent element.

\section{Free Ideals and Basic Ideals}

In this section we discuss an ideal of the algebra over a unital commutative ring $R$ that satisfies certain conditions. We recall first the definition of subalgebra and ideal in the algebra.

Definition 2.1. [2] A subalgebra of an $R$-algebra $A$ is a subset $S$ of $A$ that is both a subring of $A$ and an $R$-submodule of $A$. A two-sided ideal of $R$-algebra $A$ is a subset $I$ of $A$ that is both a two-sided ideal of $A$ and an $R$-submodule of A. Furthermore, an ideal means a two-sided ideal.

As described in the introduction, not every ideal in a free $R$-algebra $A$ has a basis because not every subalgebra has a basis too. In addition, an ideal in $A$ may be a free $R$-submodule but its basis is not in the basis of $A$. This motivates us to define an ideal which has a certain basis by following the pattern of both Definition 2.1 and definition of free $R$-algebra [2].

Definition 2.2. Let $A$ be a unital free R-algebra ( $A$ is both a unital ring and a free $R$-module). An ideal $I$ in $A$ is called a free ideal if it has a basis contained in a basis of $A$.

Note that the algebra intended in this paper is always unital algebra. Furthermore, a unital free $R$-algebra is simply written as a free $R$-algebra. It is straightforward that a free $R$-algebra $A$ generated by the basis $X$ can be denoted as $A=\langle X\rangle$, where $\langle X\rangle=\left\{\sum_{i=1}^{m} r_{i} x_{i}: r_{i} \in R, x_{i} \in X, m \in \mathbb{N}\right\}$. On the other hand, if $A$ is a unital ring, then for any $p \in A$ we can define an ideal generated by $p$, namely, $(p)=\left\{\sum_{j} a_{j} p b_{j}: a_{j}, b_{j} \in A\right\}$. Then $(p)$ is an ideal and also an $R$-submodule in $A$. We should note that the term generated in the algebra $A$ as $R$-module is different from that one in ring $A$. Consider the following example:

Example 2.3. Let $M_{2}\left(\mathbb{Z}_{6}\right)=\left\{\left(\begin{array}{ll}a & b \\ c & d\end{array}\right): a, b, c, d \in \mathbb{Z}_{6}\right\}$ is a free $\mathbb{Z}_{6}$-algebra with a basis $X=\left\{\left(\begin{array}{ll}1 & 0 \\ 0 & 0\end{array}\right),\left(\begin{array}{ll}0 & 0 \\ 0 & 1\end{array}\right),\left(\begin{array}{ll}0 & 1 \\ 0 & 0\end{array}\right),\left(\begin{array}{ll}0 & 0 \\ 1 & 0\end{array}\right)\right\}$. It seems obvious that:

1. $\left\langle\left(\begin{array}{ll}0 & 0 \\ 0 & 1\end{array}\right)\right\rangle=\left\{\left(\begin{array}{ll}0 & 0 \\ 0 & d\end{array}\right): d \in \mathbb{Z}_{6}\right\}$ is a free $\mathbb{Z}_{6}$-subalgebra, but is not an ideal in $M_{2}\left(\mathbb{Z}_{6}\right)$. However,

$\left(\left(\begin{array}{ll}0 & 0 \\ 0 & 1\end{array}\right)\right)=\left\{\left(\begin{array}{ll}a & b \\ c & d\end{array}\right)\left(\begin{array}{ll}0 & 0 \\ 0 & 1\end{array}\right)\left(\begin{array}{ll}p & q \\ r & s\end{array}\right):\left(\begin{array}{ll}a & b \\ c & d\end{array}\right),\left(\begin{array}{ll}p & q \\ r & s\end{array}\right) \in M_{2}\left(\mathbb{Z}_{6}\right)\right\}=M_{2}\left(\mathbb{Z}_{6}\right)$

is a free ideal. Therefore, $\left\langle\left(\begin{array}{ll}0 & 0 \\ 0 & 1\end{array}\right)\right\rangle \neq\left(\left(\begin{array}{ll}0 & 0 \\ 0 & 1\end{array}\right)\right)$. 
2. $\left(\left(\begin{array}{ll}0 & 0 \\ 0 & 2\end{array}\right)\right)=\left\{\left(\begin{array}{ll}a & b \\ c & d\end{array}\right)\left(\begin{array}{ll}0 & 0 \\ 0 & 2\end{array}\right)\left(\begin{array}{ll}p & q \\ r & s\end{array}\right):\left(\begin{array}{ll}a & b \\ c & d\end{array}\right),\left(\begin{array}{ll}p & q \\ r & s\end{array}\right) \in M_{2}\left(\mathbb{Z}_{6}\right)\right\}=M_{2}\left(2 \mathbb{Z}_{6}\right)$ is an ideal in $M_{2}\left(\mathbb{Z}_{6}\right)$ but not a free ideal.

Consider the above example, and suppose $I=\left(\left(\begin{array}{ll}0 & 0 \\ 0 & 1\end{array}\right)\right) ; J=\left(\left(\begin{array}{ll}0 & 0 \\ 0 & 2\end{array}\right)\right)$. Then for any non-zero $r \in \mathbb{Z}_{6}, y \in Y, r y \in I$ implies $y \in I$, where $Y$ be any basis of $M_{2}\left(\mathbb{Z}_{6}\right)$. However, there exist $4 \in \mathbb{Z}_{6},\left(\begin{array}{ll}0 & 0 \\ 0 & 1\end{array}\right) \in X$, such that $4\left(\begin{array}{ll}0 & 0 \\ 0 & 1\end{array}\right)=$ $\left(\begin{array}{ll}0 & 0 \\ 0 & 4\end{array}\right) \in J$, but $\left(\begin{array}{ll}0 & 0 \\ 0 & 1\end{array}\right) \notin J$. Compare these cases with any ideal $I^{\prime}$ in the $K$ algebra $A^{\prime}$ with a basis $X$. The ideal $I^{\prime}$ always satisfies the following condition: if $k x \in I^{\prime}$ then $x \in I^{\prime}$, for every non-zero $k \in K$, and every $x \in X$. This gives us an idea to define an ideal in a free $R$-algebra that satisfies the condition.

Definition 2.4. Let $A$ be a free $R$-algebra. An ideal $I$ in $A$ is called a basic ideal if for every non-zero $r \in R$, and every $x \in X, r x \in I$ then $x \in I$, for any basis $X \subset A$.

It is well known that for every $k \in K \backslash\{0\}$, and for every $x \in X, k x \neq 0$, where $X$ be any basis of the $K$-algebra. The following lemma shows that this property is satisfied in free $R$-algebras. It is very useful for discussing the properties of basic ideal in the free $R$-algebra.

Lemma 2.5. Let $X$ be a basis of free $R$-algebra $A$. Then $r x \neq 0$, for every non-zero $r \in R, x \in X$.

Proof. It is clear from the definition of basis $X$ that $X$ is linearly independent, that it is, if $r x=0$ then $r=0$ for every $x \in X$.

According to Definition 2.4, any free $R$-algebra is a trivial basic ideal. In addition, $\{0\}$ is always a basic ideal in any free $R$-algebra based on Lemma 2.5. The conclusion of this description is stated in the following corollary.

Corollary 2.6. For any free $R$-algebra $A$, both ideal $\{0\}$ and $A$ are basic ideals.

Besides the above idea, Definition 2.4 also refers to the definition of basic ideal in Leavitt path algebras over a unital commutative ring. This basic ideal characterized the basically simple Leavitt path algebras [4]. Recall that a Leavitt path algebra over a unital commutative ring is basically simple if the only basic ideals are $\{0\}$ and itself. Similarly, a free $R$-algebra $A$ is basically simple if the only basic ideals are $\{0\}$ and $A$.

Furthermore, we will investigate relations between basic ideals and free ideals in free $R$-algebras. For every element in any basis of the free algebra is a generator of a basic ideal as a submodule, if it is in the ideal. This property is stated in the following lemma.

Lemma 2.7. Let $I$ be a basic ideal of the free $R$-algebra $A$. Let $X$ be an arbitrary basis of $A$. Then 
(1) For every $x \in X, x \in I$ if and only if $x$ is a generator of $I$ as an $R$ submodule.

(2) For every non zero $r_{i} \in R$ and for every $x_{i} \in X$, with $i=1,2, \ldots, n$, for some $n \in \mathbb{N}, \sum_{i=1}^{n} r_{i} x_{i} \in I$, then $x_{i} \in I$ for every $i=1,2, \ldots, n$.

Proof. Take any basis $X$ of $A$. Let $x \in X$ such that $x \in I$. Suppose that $x$ is not a generator of $I$ as an $R$-submodule. Then there is a non zero $c \in R$ such that $c x \notin I$. Since $I$ is basic then $x \notin I$, which is a contradiction. Hence, $x$ is a generator. It is easy to prove the converse. Furthermore, we use the first part of this lemma to prove the second part.

Lemma 2.7 leads us to prove that the free ideal is a necessary and sufficient condition of the basic ideal. This is an important result stated by the following theorem.

Theorem 2.8. Let $A$ be a free $R$-algebra and ideal $I \subseteq A$. Then $I$ is a basic ideal if only if $I$ is a free ideal.

Proof. It is clear that the trivial basic ideals are free. Let $X$ be any basis of $A$. Form a subset $H_{X}=\{h \in X \mid r h \in I, \forall r \in R \backslash\{0\}\}=X \cap I$. The first, we will show that $I=\{0\}$, if $H_{X}=\emptyset$ for any basis $X$. It means that $x \notin I$ for every $x \in X$. Suppose that $I \neq\{0\}$, then there is a non zero $a=\sum_{i=1}^{n} r_{i} x_{i} \in I$ for some $r_{i} \in R, x_{i} \in X$. Hence, there exist $r_{i} \in R, x_{i} \in X$, such that $\sum_{i=1}^{n} r_{i} x_{i} \in I$ and $x_{i} \notin I$. Based on Lemma 2.7, $I$ is not basic, a contradiction.

Furthermore, if $\{0\} \neq I \subsetneq A$ then there is a basis $Y$ of $A$ such that $H_{Y}=$ $Y \cap I \neq \emptyset$. Suppose that $I \neq\left\langle H_{Y}\right\rangle$ for every basis $Y$. Then there is a non zero $b \in I$ and $b \neq \sum_{i=1}^{k} c_{i} h_{i}$ for every $c_{i} \in R, h_{i} \in H_{Y}$. Consequently, $b=\sum_{j=1}^{m} r_{j} y_{j}$ for some $r_{j} \in R, y_{j} \in Y$, but $y_{j} \notin I$, for every basis $Y$. According to Lemma 2.7, $I$ is not basic, a contradiction. Hence, $I=\left\langle H_{Y}\right\rangle$ is a free ideal with a basis $H_{Y} \subseteq Y$, for a basis $Y$ of $A$. We can proof the converse using Definition 2.4, directly.

The description of Example 2.3 shows that an ideal generated by one element is not always a basic ideal in a free $R$-algebra. The following lemma states the sufficient condition of an ideal generated by one element that is a basic ideal.

Lemma 2.9. Let $A$ be a free $R$-algebra with a basis $X$. Then an ideal generated by any $h \in X$, ie. $(h)=\left\{\sum_{i} a_{i} h b_{i}: a_{i}, b_{i} \in A\right\}$ is a basic ideal.

Proof. Let $h \in X$. Then $h \in(h)$ for $h=1_{A} h 1_{A}$. Let $Y$ be any basis of $A$. Take any non zero $r \in R, y \in Y$ such that $r y \in(h)$. Then $r y=\sum_{i} a_{i} h b_{i}$, for some $a_{i}, b_{i} \in A$. Suppose that $y$ is not a generator of $(h)$ as an $R$-submodule of $A$. Then $r y=\sum_{i} a_{i} h b_{i}=\sum_{i} \sum_{y \neq y_{i j}} r_{i j} y_{i j}$ for some $r_{i j} \in R, y_{i j} \in Y$. We have $Y$ is not linearly independent, a contradiction. Since $y$ is a generator of $(h)$ as an $R$-submodule, then $y \in(h)$. Hence, $(h)$ is a basic ideal. 
A free ideal in a free $R$-algebra will play a role in the classification of the basic ideal that will characterize the free $R$-algebra. One of the characterizations of free $R$-algebras is the basically semisimple algebra. The definition of minimal basic ideal is needed to characterize that algebra. This characterization is the focus of this paper and will be described in the next section.

\section{Basically Semisimple Free Algebras over a Commutative Ring}

Let $A$ be a free $R$-algebra, then $A$ may contain a zero divisor. Similarly, as $A$ an $R$-module, the multiplication of non-zero elements in $R$ with non-zero elements in $A$ may be zero in $A$. In this notions, we will define annihilators of the ring $A$ and the $R$-module $A$ which are denoted slightly different. The annihilators and its properties are useful to discuss the theorem which is a main result of this research.

Definition 3.1. [7] Let $A$ be an R-algebra and a non empty subset $S \subset A$. Then:

(i) The left, right and (two-sided) annihilator of $S$ in $A$, respectively denoted and defined as,

$$
\begin{gathered}
A n n^{l}(S)=\{a \in A: a s=0, \forall s \in S\} \\
A n n^{r}(S)=\{a \in A: s a=0, \forall s \in S\} \\
\operatorname{Ann}(S)=\{a \in A: a s=0=s a, \forall s \in S\}=A n n^{l}(S) \cap A n n^{r}(S)
\end{gathered}
$$

(ii) The annihilator of $S$ in $R$-module $A$ denoted and defined as,

$$
A n n_{R}(S)=\{r \in R: r s=0, \forall s \in S\}
$$

We can easily show that the left, right, and two-sided annihilator of $S$ in $A$ is a left, a right, and a (two-sided) ideal in $A$, respectively. Similarly, the annihilator of $S$ in $R$-module $A$ is an ideal of $R[7]$. However, the annihilators are not necessarily a basic ideal. Consider the following example:

Example 3.2. Let $M=\left\{(a, b): a, b \in \mathbb{Z}_{6}\right\}$ be a free $\mathbb{Z}_{6}$-algebra. Take two ideals in $M$ :

$$
M^{\prime}=\left\{(p, q): p, q \in 2 \mathbb{Z}_{6}\right\} ; \quad M^{\prime \prime}=\left\{(a, 0): a \in \mathbb{Z}_{6}\right\} .
$$

Then $\operatorname{Ann}^{l}\left(M^{\prime}\right)=\left\{(u, v): u, v \in 3 \mathbb{Z}_{6}\right\}=A n n^{r}\left(M^{\prime}\right)$ is not a basic ideal, but $A n n^{l}\left(M^{\prime \prime}\right)=\left\{(0, b): b \in \mathbb{Z}_{6}\right\}=A n n^{r}\left(M^{\prime \prime}\right)$ is a basic ideal. Furthermore, if we take a central idempotent $(0,1) \in M$ then $\operatorname{Ann}(\{(1,0)\})=\left\{(0, b): b \in \mathbb{Z}_{6}\right\}$ is a basic ideal.

The above cases inspire us to investigate the properties of annihilators in $A$. The following proposition would state a sufficient condition of annihilator of a subset in the free $R$-algebra as a basic ideal.

Proposition 3.3. Let $A$ be a free R-algebra and $I$ a non empty subset of $A$. If I is a basic ideal then $\operatorname{Ann}^{l}(I), \operatorname{Ann}^{r}(I)$ and $A n n(I)$ is a basic left, right and (two-sided) ideal in $A$ respectively. 
Proof. We only prove that $A n n^{l}(I)$ is a basic left ideal, and the others can be shown similarly. Based on Proposition 2.8, $I$ is a free ideal with a basis $H \subseteq Y$ where $Y$ is a basis of $A$. Let $X$ be any basis of free $R$-algebra $A$. For every non-zero $r \in R, x \in X$ such that $r x \in A n n^{l}(I)$, we have $(r x) u=r(x u)=0$, for any $u \in I$. Since $x \in X, r \neq 0$, by Lemma $2.5, x \neq 0$ and $r x \neq 0$. We will show that $x u=0$, as follows:

Suppose $x u \neq 0$, for some $u \in I$. We have $x u \in I$ and $x u=\sum_{i=1}^{k} r_{i} h_{i} \neq 0$, where $r_{i} \in R, h_{i} \in H$. Then there is $m$, with $1 \leq m \leq k$ such that $r_{m} h_{m} \neq 0$ and $r r_{m} h_{m} \neq 0$ for some $r \in R$. We obtain the existence of $u \in I$ such that $r x u=$ $\sum_{i=1}^{k} r r_{i} h_{i} \neq 0$. It is a contradiction to $(r x) u=0$, for every $u \in I$. Thus, $x u=0$, so that $x \in A n n^{l}(I)$. In other words, $A n n^{l}(I)$ is a basic left ideal in $A$.

A minimal basic ideal in Leavitt path algebra that characterizes the basically semisimple algebra has been defined by $([5],[6])$. This definition is based on the definition of the semisimple ring $([3],[7])$ and is motivated by the definition of basically simple Leavitt path algebras over a unital commutative ring [4]. Similarly, we can define a minimal basic ideal and a basically semisimple free $R$-algebra.

Definition 3.4. Let $A$ be a free $R$-algebra and $I$ a basic ideal in $A$. Then $I$ is a minimal basic ideal if it does not contain non-zero basic ideal other than itself. A is basically semisimple if it is a direct sum of minimal basic ideals in A.

In the free $\mathbb{Z}_{6}$-algebras (see Example 2.3 and 3.2), both $M_{2}\left(\mathbb{Z}_{6}\right)$ and $M$ are basically semisimple. Since $M_{2}\left(\mathbb{Z}_{6}\right)$ is basically simple, automatically it is basically semisimple. Although $M$ is not basically simple, but $M=M " \oplus \operatorname{Ann}(\{(1,0)\})$, in which $M$ " and $\operatorname{Ann}(\{(1,0)\})$ are minimal basic ideals in $M$. The free algebra $M_{2}\left(\mathbb{Z}_{6}\right)$ and $M$ can be represented by a graph. They are isomorphic to Leavitt path algebra on a graph with two vertices. A graph of $M_{2}\left(\mathbb{Z}_{6}\right)$ has one edge and a graph of $M$ has no edges [6].

However, sometimes we could not easily show whether a free $R$-algebra is basically semisimple or not. Wisbauer [7] characterized a semisimple ring $A$ with an identity by $A$ is a finite sum of minimal ideals, or every ideal is a direct summand of $A$, and equivalently, $A$ is generated by a central idempotent element. In fact, the theorem can be implied in basically semisimple free $R$-algebra by replacing basic ideals into ideals in the theorem. The following is a main result in this paper.

Theorem 3.5. Let $A$ be a free R-algebra. The following properties are equivalent:

(a) A is basically semisimple,

(b) A is a finite sum of minimal basic ideals,

(c) Every basic ideal is a direct summand of $A$,

(d) Every basic ideal of $A$ is generated by a central idempotent element.

Proof. Let $A$ be a free $R$-algebra with a basis $X$.

$(a \Rightarrow b) A$ is basically semisimple, namely, $A=\oplus I_{t}$, where $I_{t}$ be the minimal basic ideal in $A$ and $\Lambda$ is an index set. Then $A=\sum_{t \in \Lambda}^{t \in \Lambda} I_{t}$, where $I_{t_{i}} \cap \sum_{\substack{t_{j} \in \Lambda \\ t_{i} \neq t_{j}}} I_{t_{j}}=\{0\}$. 
Since $1_{A} \in A$, then $1_{A}=\sum_{i=1}^{k} r_{i} x_{i}$, with $r_{i} x_{i} \in I_{t_{i}}$ for some $r_{i} \in R, x_{i} \in X$ and $t_{i} \in \Lambda$. Furthermore, for every monomial $y \in A$,

$$
y=y 1_{A}=y \sum_{i=1}^{k} r_{i} x_{i}=\sum_{i=1}^{k} r_{i} y x_{i}
$$

Thus $A=\sum_{i=1}^{k} I_{t_{i}}$ that is a finite sum of minimal basic ideals.

$(b \Rightarrow a)$ Suppose $J_{1}, J_{2}, \ldots, J_{m}$ are minimal basic ideals in $A$, such that $A=\sum_{i=1}^{m} J_{i}$. We will prove that $A$ is basically semisimple, as follows.

If $J_{1} \cap \sum_{i=2}^{m} J_{i}=P \neq\{0\}$, then ideal $P \subseteq J_{1}$. Since $J_{1}$ is a minimal basic ideal, we have $J_{1} \subseteq P$. It means that $J_{1} \cap \sum_{i=2}^{m} J_{i}=J_{1}$, so that $J_{1}$ has no effect on the representation of $A$. But, $J_{1} \cap \sum_{i=2}^{m} J_{i}=\{0\}$ then $A=J_{1} \oplus \sum_{i=2}^{m} J_{i}$. Note the remainder sum $\sum_{i=2}^{m} J_{i}$, and by the same reason that $J_{2}$ has no effect on the representation of $A$ or $\sum_{i=2}^{m} J_{i}=J_{2} \oplus \sum_{i=3}^{m} J_{i}$. The process is continued for the remainder of the next sum, and remove all the basic ideal that has no effect on the representation of $A$. We obtain representation of $A$ as the sum of remaining minimal basic ideals. Because every remaining minimal basic ideal which intersects to the remainder of the sum is $\{0\}$, then the representation of $A$ is the direct sum. Thus, $A$ is basically semisimple.

$(c \Rightarrow d)$ For every basic ideal $I \subset A$, by hypothesis there is a basic ideal $J \subset A$ such that $A=I \oplus J$. We will show that $I$ is generated by a central idempotent element. Since $A$ has an identity $1_{A}$, then $1_{A}=a+b$ with $a \in I, b \in J$. So we obtain: $a=a 1_{A}=a(a+b)=a^{2}+a b$ or $a b=a-a^{2}$. In addition, $I$ and $J$ are ideals and $A=I \oplus J$ then $a b \in I \cap J=\{0\}$. It means that $a b=a-a^{2}=0$ or $a^{2}=a$. Thus $a$ is idempotent. Furthermore, for every $x \in I$, then $x=x 1_{A}=x(a+b)=x a+x b$ or $x b=x-x a \in I \cap J=\{0\}$, since $x b \in I, x b \in J$. Hence, $x=x a$ or $I$ is generated by $a$. In the other hand, for every monomial $y \in A, y=y a+y b$ and $y=a y+b y$ then $y a, a y \in I, y b, b y \in J$. Then it should be $a y=y a$ or $a$ is a central idempotent, because of $A=I \oplus J$. Thus, $I=(a)$, in which $a$ is a central idempotent.

$(d \Rightarrow c)$ For every basic ideal $I \subset A, I=(i)$, whence $i$ is a central idempotent. We will find a basic ideal $J$ such that $A=I \oplus J$, as follow :

We have $i \in I$ and $i$ is a central idempotent. Then for any $a \in A, a=a i+a-a i$, in which $a i \in I$ and $a-a i \in \operatorname{Ann}(i)$, so $a \in I+\operatorname{Ann}(i)$. In the other hand, for every $p \in I \cap A n n(i), p=y i$ for some $y \in A$ and $p i=0$. It implies that $p=y i=y i^{2}=(y i) i=p i=0$, then $I \cap \operatorname{Ann}(i)=\{0\}$. Hence, $A=I \oplus \operatorname{Ann}(i)$.

Furthermore, we will prove that $\operatorname{Ann}(i)$ is a basic ideal. Since $i$ is central idempotent, then $\operatorname{Ann}(i)=\{a \in A: a i=0=i a\}$. If $i=0$, then it is clear that $A n n(i)=A$ is a basic ideal for Corollary 2.6. If $i \neq 0$ then $1_{A} i 1_{A}=i \in I$ and $i \notin A n n(i)$. For every non-zero $r \in R, x \in X$, such that $r x \in A n n(i)$ then we have $r x \neq 0$ from Lemma 2.5. Since $r x \in A n n(i)$ and $A=I \oplus A n n(i)$ then $r x \notin I$. From Proposition 2.8, ideal $I$ is free with the basis $H=I \cap X$, so we have $x \notin I$. It should be $x \in \operatorname{Ann}(i)$. Hence, $\operatorname{Ann}(i)$ is a basic ideal.

$(a \Rightarrow c)$ Let $A$ be basically semisimple. Based on the proof of ( $a \Leftrightarrow b)$, we have $A=J_{1} \oplus J_{2} \oplus \ldots \oplus J_{m}$ with $J_{1}, J_{2}, \ldots, J_{m}$ are minimal basic ideals in $A$. For any 
basic ideal $I \subset A$, we will investigate that there exists a basic ideal $J \subset A$ such that $A=I \oplus J$. We have $J_{i} \cap I=J_{i}$ or $J_{i} \cap I=\{0\}, \forall i=1,2, \ldots, m$ by hypothesis that $J_{1}, J_{2}, \ldots, J_{m}$ are minimal basic ideals. For every $i=1,2, \ldots, m$, if $J_{i} \cap I=J_{i}$ then $J_{i} \subseteq I$. It means that $A=J_{1} \oplus J_{2} \oplus \ldots \oplus J_{m} \subseteq I$, thus $I=A$. In this case, just take the basic ideal $J=\{0\}$. If not, it means that there is a minimal basic ideal $J_{n_{1}}, 1 \leq n_{1} \leq m$ such that $J_{n_{1}} \cap I=\{0\}$. Further, look at another minimal basic ideal. If there exists here, select a minimal basic ideal $J_{n_{2}}, 1 \leq n_{2} \leq m, n_{2} \neq n_{1}$ such that $\left(J_{n_{1}} \oplus J_{n_{2}}\right) \cap I=\{0\}$. The process is continued such that we obtain no more basic ideal, in which the intersection of the basic ideal and ideal $I$ equals to $\{0\}$. Finally, this results in the basic ideals $J_{n_{1}}, J_{n_{2}}, \ldots, J_{n_{s}}$, with $s \leq m$, and $1 \leq n_{1}, n_{2}, \ldots, n_{s} \leq m$, such that $\left(J_{n_{1}} \oplus J_{n_{2}} \oplus \ldots \oplus J_{n_{s}}\right) \cap I=\{0\}$. Furthermore, let $\boldsymbol{I}=\left(J_{n_{1}} \oplus J_{n_{2}} \oplus \ldots \oplus J_{n_{s}}\right) \oplus I$, we will show $\boldsymbol{I}=A$ and we sufficiently prove $A \subseteq I$, as follows :

It is clear that $J_{n_{1}}, J_{n_{2}}, \ldots J_{n_{s}} \subseteq \mathcal{I}$. Suppose there is a minimal basic ideal $J_{k}$ with $k \neq n_{i}, i=1,2, \ldots, s$ such that $J_{k} \cap \mathbb{I}=\{0\}$, then $\left(J_{n_{1}} \oplus J_{n_{2}} \oplus \ldots \oplus J_{n_{s}} \oplus J_{k}\right) \cap I=\{0\}$, which is a contradiction with the election of $J_{n_{1}}, J_{n_{2}}, \ldots, J_{n_{s}}$. Thus, it should be $J_{k} \cap \boldsymbol{I}=J_{k}, \forall k, 1 \leq k \leq m$, and $k \neq n_{i}, i=1,2, \ldots, s$. It means that $\boldsymbol{I}=A$, since $A=J_{1} \oplus J_{2} \oplus \ldots \oplus J_{m} \subseteq$ I. In this case, it is true that $A=I \oplus J$ by taking basic ideal $J=J_{n_{1}} \oplus J_{n_{2}} \oplus \ldots \oplus J_{n_{s}}$.

$(c \Rightarrow a)$ We will prove that $A$ is basically semisimple, by hypothesis for every basic ideal $I \subset A$, there exists a basic ideal $J$ such that $A=I \oplus J$.

At first, we will show that any basic ideal generated by a non-zero central idempotent $p$, namely, $(p)$ contains a minimal basic ideal. Consider that

$$
\Im=\{I \subset(p) \text { : basic ideal } I \subset A, p \notin I\}
$$

is the family of basic ideals in $A$ that is contained in $(p)$ and contains no $p$. According to Zorn's lemma, there is a maximal $Q$ in $\Im$. Based on the proof of $(c \Leftrightarrow d)$, we obtain $Q=(q)$ for a central idempotent $q$, and $(p)=(q) \oplus(p-p q)$. We will see that $(p-p q)$ is a minimal basic ideal, as follows:

Suppose that $(p-p q)$ contains a non trivial basic ideal $N$ with $N \neq(p-p q)$ then $p-p q \notin N$. Consequently, $p \notin(q)+N$ and since $(q) \subset(q)+N \subset(p)$ then $(q)+N \in \Im$. This contradicts $Q=(q)$ as a maximal element in $\Im$. Hence, $(p-p q)$ is a minimal basic ideal. Finally, consider $M$ is a sum of all minimal basic ideals in $A$. By hypothesis, there is basic ideal $C$ in $A$ such that $A=M \oplus C$. Because $C$ contains no minimal basic ideals then $C=\{0\}$, so that $A=M$. Thus, $A$ is a direct sum of minimal basic ideals or $A$ is basically semisimple.

Example 3.6. Consider $T=(x, y: x y=1 \neq y x)$ is Toeplitz algebra over unital commutative ring $R$. The algebra is a ring generated by two variables $x$ and $y$ that satisfy $x y=1 \neq y x$. If the algebra $T$ is viewed as $R$-module then its generators are infinite, which will be more easily understood by the approach of Leavitt path algebra with the following graph:

This time it is not so straightforward that the maps $x \mapsto e^{*}+f^{*}$ and $y \mapsto e+f$ yield an isomorphism between $T$ and leavitt path algebra [1]. It is due to all of vertices 
and edges are representation of element in $T$, namely,

$$
v \mapsto y x ; w \mapsto x y-y x=1-y x ; e \mapsto y^{2} x ; e^{*} \mapsto y x^{2} ; f \mapsto y-y^{2} x ; f^{*} \mapsto x-y x^{2}
$$

Based on the graph, we have non-zero idempotent elements in $T$, which are a part of a basis of $T$, including $x y=1, y x, 1-y x$, and $y x-y^{2} x^{2}$. Furthermore, according to Lemma 2.9 we have $I=(1-y x)$ is a basic ideal generated by $1-y x$. However, $I=(1-y x) \neq T$ because there is a monomial $y^{2} x \in T$ and $y^{2} x \notin I$. Moreover, $1-y x$ is idempotent but not central idempotent, because there is $y \in T$ whence $y(1-y x)=y-y^{2} x \neq(1-y x) y=0$. Hence, there is a basic ideal in $T$ that is not generated by a central idempotent element. Then based on the Theorem 3.5, $T=(x, y: x y=1 \neq y x)$ is not basically semisimple.

\section{Concluding Remarks}

Based on the above discussion, we conclude many significant remarks. One of them is that a free ideal is a necessary and sufficient condition of an ideal in a free algebra over ring to be a basic ideal. In addition, an ideal generated by one element in a free algebra over ring is a basic ideal if the element is in its basis. The two significant results are useful to discuss Theorem 3.5. This theorem is a main point of this paper. In the future, we will discuss basically (semi) prime free algebras over ring and its properties that characterized by (semi) prime basic ideal.

Acknowledgement. The authors are grateful to Prof. Bas Edixhoven of Leiden University who has given corrections to the definition of the free ideal and some inputs to this article. The authors also thank to the anonymous reviewers for their comments and suggestions.

\section{REFERENCES}

[1] Abrams, G., Aranda Pino, G. Perera, F., and Molina, S.M., "Chain conditions for Leavitt path algebras", Forum Math. (To appear.)

[2] Grillet, P.A., Abstract Algebra, Graduated Texts in Mathematics, Spinger-Verlag, New York, 2007.

[3] Lam, T.Y., A First Course in Noncommutative Rings, Springer-Verlag, New York, 1991.

[4] Tomforde, M., "Leavitt Path Algebras with Coefficient In A Commutative Ring", J. Pure Appl. Algebra, 215 (2011), 471-484.

[5] Wardati, K., Wijayanti, I.E. and Wahyuni, S., "On Basic Ideal in Leavitt Path Algebra" (In Indonesia), Proceedings of Mathematical National Conference XVI, Mathematics Departement, Padjadjaran University, Bandung, Indonesia, ISBN 978-602-19590-2-2, (2012), $75-84$.

[6] Wardati, K., Wijayanti, I.E. and Wahyuni, S., "Basically Semisimple of Leavitt Path Algebra on Acyclic Finite Graph" (In Indonesia), Presented in "Seminar Nasional dan Workshop Aljabar dan Pembelajarannya", Mathematics Departement, Universitas Negeri Malang, Indonesia, 2013. 
[7] Wisbauer, R., Foundations of Module and Ring Theory, Gordon and Breach Publishers, 1991. 